Cite this: Soft Matter, 2013, 9, 11490

\title{
Elastic properties of cells in the context of confluent cell monolayers: impact of tension and surface area regulation $\dagger$
}

\author{
Anna Pietuch, ${ }^{a}$ Bastian Rouven Brückner, ${ }^{a}$ Tamir Fine, ${ }^{b}$ Ingo Mey ${ }^{c}$ \\ and Andreas Janshoff*a
}

Epithelial cells usually form a dense continuous cobblestone-like sheet that is frequently exposed to a variety of mechanical challenges encompassing osmotic stress and external forces. The response to external forces was investigated and the question of how individual polar epithelial cells organized in confluent monolayers respond to pharmaceutical stimuli targeting the key players of cellular mechanics was answered. In particular, we ask how epithelial cells respond to changes in cortical and membrane tension by surface area regulation if challenged by diverse chemical and mechanical cues. Here, a tension-based model is used that allows capturing the relevant modes of cell deformation. Together with independent measurements of membrane tension, cortical tension and excess surface area of confluent MDCK II cells it is possible to draw a mechanistic picture of how confluent cells respond to mechanical stimuli in general. Changes in tension are provoked by external stimuli directed towards the contractile actomyosin cortex (cytochalasin D, blebbistatin), and changes in the excess surface area are produced by cholesterol extraction (methyl- $\beta$-cyclodextrin) or inhibition of dynamin (dynasore). A combination of AFM-indentation experiments with membrane-tether pulling at the same position allowed us to simultaneously monitor changes in membrane tension, cortical tension and excess surface area. Generally, we observed that removing or producing excess surface area of the plasma membrane readily adjusts membrane tension that is pivotal for the mechanical response of confluent cells. We found that isolated apical membranes from confluent MDCK II monolayers display similar mechanical properties as the apical side of living MDCK II cells in a confluent monolayer confirming that membrane mechanics in conjunction with cytoskeletal adhesion dominates the elastic response of confluent epithelial cells at large strain.

Received 11th June 2013

Accepted 19th August 2013

DOI: 10.1039/c3sm51610e

www.rsc.org/softmatter most prominently the so-called Hertz model that describes the weak elastic deformation of a homogenous continuum with a spherical indenter in the absence of adhesion. While this model seems useful to obtain an overall bulk Young's modulus of the probed cell, it does not capture the intricate shell-like architecture of living cells and therefore might provide only a qualitative picture of the cellular stiffness. ${ }^{4-6}$

From a biological point of view, the cellular shape is formed by a membrane shell connected to an actomyosin cortex via adhesion proteins such as those belonging to the ERM (ezrinradixin-moesin)-protein family. ${ }^{7}$ The contractile nature of this active gel allows to exert a tension that naturally produces a pressure difference between the cytosol and the extracellular space driving cell deformation such as bleb formation or pseudopodia extension necessary for cell locomotion. Tinevez et al. recently described the role of cortical tension in bleb growth, which allows relating bleb formation to cellular mechanics and demonstrates how generation of blebs can be regulated during cell motility. ${ }^{8-10}$ Besides cortical tension, which dominates in suspended cells, ${ }^{8}$ plasma membrane 
tension is recognized as a major regulator of many cellular processes that involve changes in the membrane area and membrane deformation including cell migration, ${ }^{11}$ mitosis, ${ }^{12}$ endocytosis, ${ }^{13}$ exocytosis, ${ }^{14}$ membrane repair, ${ }^{15}$ osmoregulation, ${ }^{16}$ and cell spreading. ${ }^{17-19}$ As the plasma membrane is largely inextensible and cannot bear large strains due to its liquid-crystalline nature limiting critical area dilatation to 2$3 \%,{ }^{20}$ rapid adjustments of surface area are required to avoid lysis of the membrane since membrane tension can quickly grow at large strains. Surface area regulation requires the existence of a dynamic membrane reservoir, which can be readily recruited if the cell changes its volume or shape as it is important in the aforementioned biological processes associated with cell division, migration, and contractility. Excess of plasma membrane is usually stored in membrane folds such as caveolae, membrane protrusions or associated vesicles. For instance, in adipocytes around $50 \%$ of the available surface area is stored in caveolae. ${ }^{21} \mathrm{An}$ increase in cell volume, for instance, as it occurs periodically in cardiomyocytes leads to an increase in membrane tension that is compensated by the loss of caveolae or other membrane reserves. ${ }^{22}$

The goal of this study was to find a mechanical model that captures the architecture of the apical membrane of confluent cells and provides access to parameters such as cortical tension, membrane tension and excess surface area from conventional force measurements with an atomic force microscope. The complex interplay between the cytoskeleton and plasma membrane requires a well-established biological model with limited perturbation from cell growth, migration, and proliferation. Here, we chose confluent Madin-Darby canine kidney cells (MDCK II) to investigate tension controlled cellular membrane area regulation. Indentation experiments combined with pulling of membrane tethers were used to assess local changes in membrane tension and excess surface area as a function of external stimuli. Our approach allows us, for the first time, to simultaneously measure cortical tension, membrane tension and changes in surface area at the same spot providing deep insight into the mechanical interplay of tension and area regulation. Chemical stimuli to provoke changes in tension and excess surface area comprise interference with the functionality of the actomyosin cortex using blebbistatin ${ }^{23,24}$ and cytochalasin $\mathrm{D},{ }^{25,26}$ arresting of endocytosis by applying the dynamin inhibitor dynasore, ${ }^{27}$ and clearing of microvilli by cholesterol-extraction from the plasma membrane with methyl$\beta$-cyclodextrin. ${ }^{28,29}$ A key experiment in this context was to determine the elastic properties of apical cell membranes derived from confluent MDCK II cell monolayers in the absence of the cell interior and devoid of the cytoskeleton. This was achieved by transferring the apical membrane of a confluent monolayer to a porous matrix allowing measurement of the elastic properties without perturbation from the cell interior. We found that pore spanning apical membranes show essentially the same elastic properties as those of living cells. This confirms our central hypothesis that membrane mechanics, i.e. excess surface area regulation in the context of membrane tension, plays a pivotal role in overall cellular mechanics of confluent epithelial cells especially at large strains.

\section{Results}

In the following section we first briefly summarize the basic physics of the mechanical model used to describe force-cycle experiments comprising indentation and tether pulling of confluent cells. Secondly, we will provide clear evidence for the validity of the proposed approach by transferring apical membranes devoid of cellular interior and cytoskeleton onto a porous mesh. Indentation experiments carried out on these pore spanning apical membranes permit us to quantify to what extent membrane mechanics contributes to the elastic response of polar cells in the context of a confluent monolayer. Eventually, we will describe the results of external stimulation with chemical cues to interfere with tension and surface area regulation.

\subsection{Mechanical behaviour of confluent epithelial cells}

The mechanical model used to describe force indentation experiments of cells in the context of a confluent monolayer relies on the assumption that the restoring force in response to indentation originates mainly from a constant isotropic tension $T$ comprising the sum of cortical and overall tension $T_{0}$ and a term describing the stretching or area dilatation of the bilayer (eqn (1)):

$$
T=T_{0}+K_{\mathrm{A}} \frac{\Delta A}{A_{0}}
$$

$\Delta A$ denotes the change in surface area related to the initial area $A_{0}$ of the computed cell. The corresponding area compressibility modulus $K_{\mathrm{A}}$ of the plasma membrane is usually in the range of $0.1 \mathrm{~N} \mathrm{~m}^{-1}$ emphasizing the inextensibility of the lipid bilayer, while that of the actin mesh is considerably smaller. ${ }^{30}$ The following argument provides reasonable values for the area compressibility modulus of the cortex: assuming the thickness of the cortex to be $1 \mu \mathrm{m}$ and the Young's modulus to be $1 \mathrm{kPa}$ we arrive at about $0.001 \mathrm{~N} \mathrm{~m}^{-1}$, which is more than 2 orders of magnitude lower than the $K_{\mathrm{A}}$ of a fluid lipid bilayer. While at low penetration depths and therefore when small strain overall tension $T_{0}$ might prevail, larger deformation resulting in stretching of the surface is governed by plasma membrane mechanics. Since the membrane is largely inextensible allowing only $2-3 \%$ of area dilatation, ${ }^{20}$ stretching of the bilayer leads in first order to a contribution described by a 2-D Hookean behaviour that adds to membrane's overall tension (eqn (1)). Therefore, the restoring force $F$ generated by the isotropic tension $T$ increases nonlinearly with indentation depth $\delta$, the distance the tip penetrates into the cell (Fig. 1C). As a consequence, stretching or area dilatation of the bilayer might dominate the elastic response at large strains. The overall tension $T_{0}$ is dominated by the cortical tension $T_{\text {act }}$ generated by active contraction of the actomyosin cortex or adhesion due to cell-cell or cell-substrate contacts. ${ }^{\mathbf{8}, 31}$ Additionally, there is a contribution from the membrane tension $T_{\mathrm{t}}$ comprising the membrane's in-plane tension $\gamma_{\mathrm{m}}$ and the tension resulting from cytoskeleton-membrane attachment sites $\gamma_{\text {ad }}$ (eqn (2) and (3)).

$$
\begin{aligned}
& T_{0}=T_{\mathrm{act}}+T_{\mathrm{t}} \\
& T_{\mathrm{t}}=\gamma_{\mathrm{m}}+\gamma_{\mathrm{ad}}
\end{aligned}
$$


A

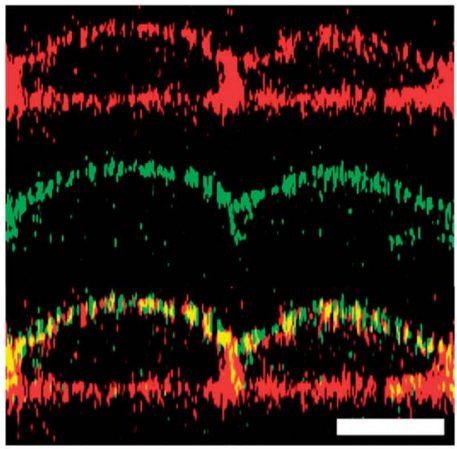

C

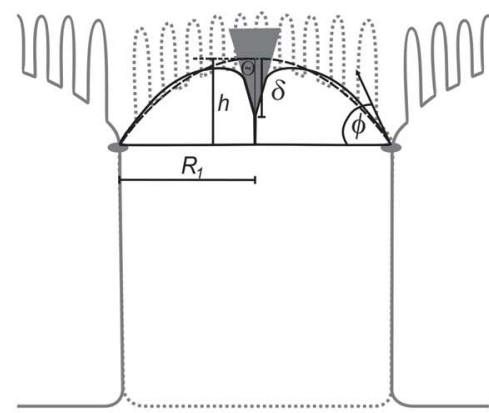

B
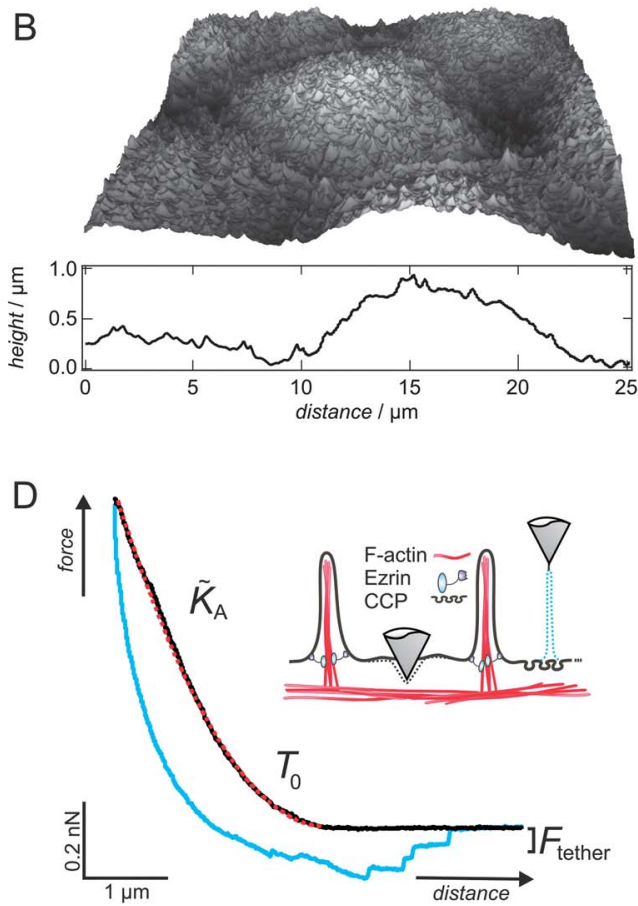

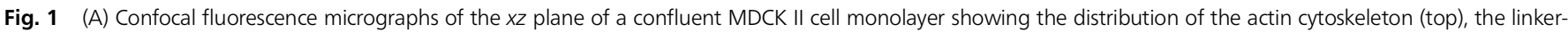

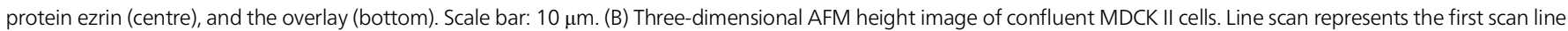

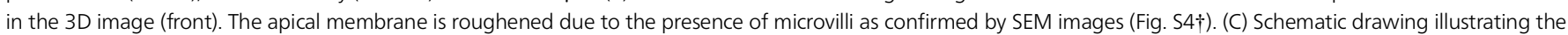

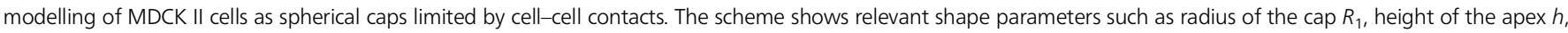

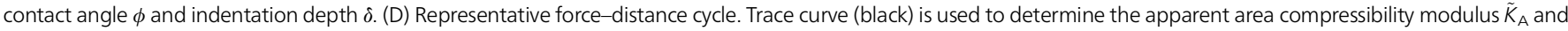

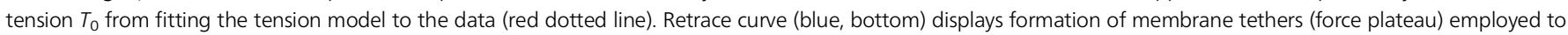
determine tension $T_{\mathrm{t}}$ from eqn (5). The inset of (D) illustrates what kind of information is gathered from a complete force cycle (CCP: clathrin coated pits).

If, however, the real surface area of the cell is larger than the projected or geometrical one $A_{0}$, due to wrinkles and folds of the membrane such as protrusions or invaginations on smaller length scales, a so-called apparent area compressibility modulus $\tilde{K}_{\mathrm{A}}$ replaces $K_{\mathrm{A}}$. A large excess of surface area $A_{\mathrm{ex}}$ generates a smaller apparent area compressibility modulus $\tilde{K}_{\mathrm{A}}($ eqn (4)).

$$
\tilde{K}_{\mathrm{A}}=K_{\mathrm{A}} \frac{A_{0}}{A_{0}+A_{\mathrm{ex}}}
$$

In order to use the correct topology for the tension model we assume that the cell-cell contacts connected to the contractile actin ring serve as tension-generating boundaries justified by AFM topography images of confluent untreated cells that display cell-cell contacts as slightly elevated and more rigid ring-like structures around each cell body (Fig. 1A and B). Fig. 1A shows confocal fluorescence images of the $x z$ plane of a confluent MDCK II monolayer encompassing the distribution of the actin cytoskeleton (top), the linker-protein ezrin (middle), and the overlay (bottom). The apical surface is imaged by AFM (Fig. 1B, height image), fluorescence microscopy and scanning electron microscopy (Fig. S4†). Thereby, we arrive at a model for the apical cap illustrated in Fig. 1C. Generally, the cap is parameterized according to the model of Sen et al. (Fig. 1C) ${ }^{32}$ The exact geometry is important since the shape of a spherical cap during indentation needs to be computed in order to obtain the restoring force as a function of the indentation depth. For this purpose, atomic force microscopy imaging was used to access the radius of the cap $R_{1}$ and the contact angle $\phi$ (see Fig. $1 \mathrm{~B}$ and $\mathrm{C}$ ). These data serve as input parameters for modelling of force indentation curves (Fig. 1C). Essentially, the elastic properties and response of epithelial cells were probed by site-specific indentation experiments followed by tether pulling upon retraction of the cantilever. While indentation data provide us with the overall tension $T_{0}$, tether pulling gives access to the membrane tension $T_{\mathrm{t}}$ alone (Fig. 1D). This procedure allows us, in principle, to distinguish between membrane tension and cortical tension.

A typical force-cycle showing the indentation in black and the retraction from the contact area in blue (bottom) is shown in Fig. 1D. Assuming that bending of the membrane/cortex can be neglected ${ }^{33}$ and tension is the only source of the restoring force experienced in indentation experiments, we can safely apply the tension model of Sen et al. (eqn (1)) to fit our force indentation curves. $^{32}$ The model essentially assumes that tension is uniform and the curvature as well as volume remains constant during indentation. The shape of the apex parameterized by the radius of the cap and the contact angle (Fig. 1C) is computed at each indentation depth, a procedure that allows accessing the restoring force as a function of indentation depth $\delta$. Therefore, the corresponding fitting function comprises only two independent parameters, namely $T_{0}$ and $\tilde{K}_{\mathrm{A}}$. While $T_{0}$ dominates the force-indentation curve at low penetration depths, $\tilde{K}_{\mathrm{A}}$ can be 
inferred from the nonlinear part of the curve at large strains where stretching of the bilayer governs the response (Fig. 1D). For pulling tethers upon retraction, we functionalized AFM tips with concanavalin A that provides enough adhesion to extract membrane nanotubes from the apical membrane. ${ }^{34}$ From the force plateau $\left(F_{\text {tether }}\right)$ of a fully established membrane nanotube, we can calculate the membrane tension $T_{\mathrm{t}}$ (Fig. 1D and eqn (5))::35,36

$$
T_{\mathrm{t}}=\frac{F_{\text {tether }}^{2}}{8 \pi^{2} \kappa}
$$

For computing $T_{\mathrm{t}}$ we chose a bending module $\kappa$ of $2.7 \times$ $10^{-19} \mathrm{~J}$ representing fluid lipid bilayers..$^{37}$ We found by varying the pulling velocity that viscous contributions to the tether force could be neglected as a first approximation..$^{38,39}$

The consistency of our approach to explain indentation experiments by active cortical and membrane tension as the key players is checked by various experiments in which the most technical one comprises the change in indenter geometry to verify our hypothesis. Two prominent models, a conventional contact model based on Hertzian mechanics (eqn (6) and (7)) and our tension model (eqn (1)) were applied. The idea is that different indenter geometries should result in the same parameters $T_{0}$ and especially $\tilde{K}_{\mathrm{A}}$ for the same cell. The theoretical models were adapted to account for the change in indenter geometry. Experimentally we used a pyramidal and a spherical indenter on the identical cell. Fig. 2A shows two averaged force-indentation curves $(n=35)$ using a conventional pyramidal indenter (triangle) and a $2 \mu \mathrm{m}$ diameter large spherical indenter (circles) recorded on the centre of a single cell of a confluent MDCK II monolayer. Note that the pyramidal tip penetrates deeper into the cell compared to the spherical indenter at the same load.

The curves were subject to fitting of the parameters of a Sneddon model (eqn (6)) by assuming a conical indenter (green dashed line) or the original Hertz model (eqn (7)) valid for spherical indenters, respectively (orange dashed line): ${ }^{40}$

$$
\begin{gathered}
F=\frac{2 \tan \theta}{\pi} \frac{E}{(1-\nu)^{2}} \delta^{2} \\
F=\frac{4}{3} \sqrt{r_{\text {sphere }}} \frac{E}{(1-\nu)^{2}} \delta^{3 / 2}
\end{gathered}
$$

where $\theta$ describes the half opening angle of the conical indenter, $\delta$ is the indentation depth, $\nu$ is the Poisson ratio $(\nu=$ $0.5)$ and $r_{\text {sphere }}$ is the radius of the spherical indenter.

The contact models of Sneddon (eqn (6)) and Hertz (eqn (7)) contain only a single parameter to describe the mechanics of the cell, the so-called Young's modulus $E$. The validity of the contact models is inherently restricted to small indentation depths. Therefore, we limited the fitting range to the first $250 \mathrm{~nm}$ of the indentation depth. Enlarging the limit to the full range results in an unsatisfactory agreement between the fit and data (data not shown). In contrast, the tension model (red lines) providing two free parameters $\left(T_{0}\right.$ and $\left.\tilde{K}_{\mathrm{A}}\right)$ fits very well to the data within the entire penetration range and the obtained parameters are largely independent of the indenter shape. The
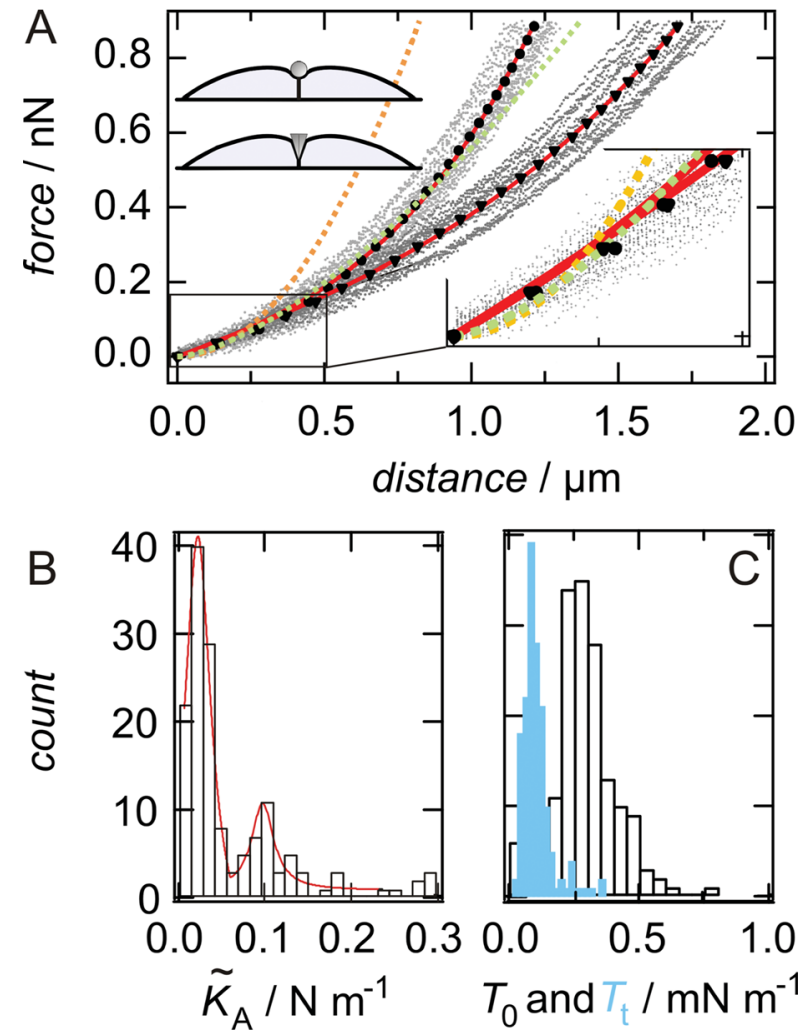

Fig. 2 Results of force cycle measurements to assess the mechanical properties of the epithelial cell line MDCK II. (A) shows the averaged force-indentation curves in an array of curves ( $n=35$ ) using a pyramidal indenter (triangle, dark gray band) and a $2 \mu \mathrm{m}$ large spherical indenter (dots, light gray band) recorded on the centre of a confluent MDCK II cell. Force curves were subject to fitting of the Sneddon model assuming a conical indenter (green dashed line) or the original Hertz model valid for spherical indenters, respectively (orange dashed line). The tension model (red lines) providing two free parameters $\left(T_{0}\right.$ and $\left.\tilde{K}_{A}\right)$ fits very well to the experimental data regardless of the indenter geometry. Insets show the corresponding computed shapes of the indented apex depending on the indenter geometry at $2.5 \mathrm{nN}$ external force. (B) Histogram of the apparent area compressibility modulus $\tilde{K}_{\mathrm{A}}$ showing a bimodal distribution $(n=160)$. The red line represents Gaussian fits of peak I and II. (C) Histogram of tension values $T_{0}$ obtained from indentation experiments $(n=160)$. The blue histogram shows tension values $T_{\mathrm{t}}$ calculated from tether pulling experiments $(n=160)$.

corresponding computed shape of the indented apex depending on the indenter geometry at $2.5 \mathrm{nN}$ external force is shown exemplarily in the inset of Fig. $2 \mathrm{~A}$ showing that the sphere does penetrate into the cell less deeply than the conical tip. Fig. 2B and $\mathrm{C}$ summarize the outcome of the fitting procedure. The force response to a conical or pyramidal indenter with identical opening angle is virtually indistinguishable.

Apart from the fact that the regression analysis of the Hertzian contact model describes the experimental data only poorly, the two contact models also fail to produce Young's moduli that are invariant of the indenter geometry. We found that the spherical indenter produces a pseudo-softer cell body by more than one order of magnitude (Table 1). This trend continues using a very large sphere ( $6 \mu \mathrm{m}$ in diameter) leading to even smaller Young's moduli of only a few Pascal (data not shown). This behaviour of the Hertzian contact model has also been observed by others but has not been understood so far. ${ }^{41}$ In 
Table 1 Results from fitting the two contact models (eqn (6) and (7)) and tension model (eqn (1)) to the averaged force curves

\begin{tabular}{lccc}
\hline & $E^{a}[\mathrm{~Pa}]$ & $T_{0}{ }^{b}\left[\mathrm{mN} \mathrm{m}^{-1}\right]$ & $\tilde{K}_{\mathrm{A}}{ }^{c}\left[\mathrm{~N} \mathrm{~m}^{-1}\right]$ \\
\hline $\begin{array}{l}\text { Spherical indenter } \\
\text { Conical indenter }\end{array}$ & $105.2 \pm 2.0$ & $0.14 \pm 0.02$ & $0.050 \pm 0.001$ \\
& $1727.1 \pm 66.8$ & $0.37 \pm 0.04$ & $0.05 \pm 0.01$ \\
${ }^{a}$ Young's modulus. ${ }^{b}$ Overall tension. ${ }^{c}$ Apparent area compressibility \\
modulus.
\end{tabular}

contrast to this ill behaviour of the Hertzian contact model, the tension model describes the experimental data over the full indentation depth very well and delivers consistent parameters that are largely invariant of indenter geometry (Table 1). Using the spherical indenter, we found an overall tension $T_{0}$, which is by a factor of 2 smaller than the tension measured by using a conical indenter, which might be attributed to a stronger adhesion of the cell to the larger indenter or bending of the cortex. The apparent area compressibility modulus is, however, identical for both types of indenters.

Fig. 2B and $\mathrm{C}$ show histograms of apparent area compressibility modulus $\tilde{K}_{\mathrm{A}}$ and tensions $T_{0}$ and $T_{\mathrm{t}}$ of a confluent MDCK II cell monolayer measured by indention and subsequent tether pulling. Following eqn (2) the active cortical tension $T_{\text {act }}\left(\bar{T}_{\text {act }}=\right.$ $\left.0.19 \pm 0.06 \mathrm{mN} \mathrm{m}^{-1}\right)$ is larger than the membrane tension $T_{\mathrm{t}}$ $\left(\bar{T}_{\text {act }}=0.08 \pm 0.04 \mathrm{mN} \mathrm{m}^{-1}\right)$ and therefore dominates the cell's response to indentation. However, it is noteworthy that the membrane tension of the confluent cells is substantial, in contrast to the membrane tension found for adherent motile cells ranging from $0.003 \mathrm{mN} \mathrm{m}^{-1}$ in neuronal growth cones $^{42}$ to $0.05 \mathrm{mN} \mathrm{m}^{-1}$ in melanoma cells. ${ }^{37}$

The apparent area compressibility modulus $\tilde{K}_{\mathrm{A}}$ of untreated MDCK II cells shows a bimodal distribution, with one maximum centred at $\tilde{K}_{\mathrm{A}}=0.02 \mathrm{~N} \mathrm{~m}^{-1}(68 \%)$ and the second one at $\tilde{K}_{\mathrm{A}}=0.1 \mathrm{~N} \mathrm{~m}^{-1}$ (peak II, 32\%) (Fig. 2B).

\subsection{Probing mechanics of isolated cell membranes}

Changes of the mechanical properties are attributed to alterations directly associated with the cortex and the plasma membrane excluding contributions from the cell interior. Direct evidence that the outer shell and particularly the plasma membrane are predominantly responsible for the mechanical response of living cells in a confluent monolayer can only be provided by probing the elasticity of isolated, planar apical cell membrane fragments derived from confluent monolayers. Previously, we established a protocol, the sandwich-cleavage approach, that allows measuring the local elastic properties of isolated apical cell membrane sheets on porous supports (pore size $1.2 \mu \mathrm{m}$, Fig. 3A-D). ${ }^{43}$ Site-specific force indentation curves in the centre of each individual pore provide a map of membrane elasticity in the absence of contributions from the cell body and also largely without interference from the cytoskeleton (Fig. 3E). Only few molecules of the cortex remain associated with the membrane fragments. The porous mesh allows us to map the site-specific area compressibility modulus of apical cell membranes neglecting the bending of the
A

B

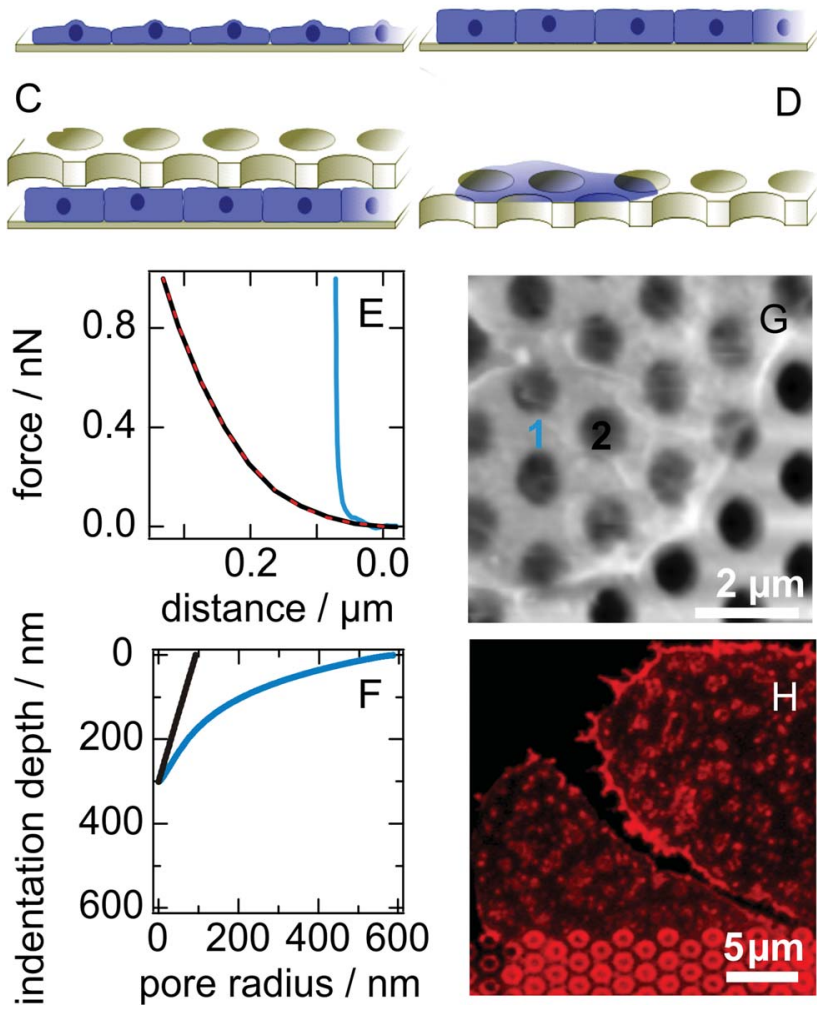

Fig. 3 (A-D) Illustration of apical membrane sheet preparation on a porous substrate. (A and B) Confluent MDCK II cells are blown up by hypoosmotic shock prior to membrane deposition. (C and D) A poly-D-lysine coated porous chip is gently pressed on the apical surface of the cells. By lifting the chip off from the cells, the apical membrane is transferred to the porous substrate. (E) Forcedistance approach curves taken on the rim (blue, right) or on a pore covered with a membrane (black, left). Red dots represent the fit according to eqn (8). (F) Calculated shape (blue line) of a membrane indented by a conical or pyramidal indenter (black line) assuming a lateral tension $T_{0}$ of $0.16 \mathrm{mN} \mathrm{m}^{-1}$ and a bending modulus $\kappa$ of $25 k_{\mathrm{B}} T$. The shape of the membrane is essentially identical to that of a point-like indenter justifying the use of eqn (8) to fit the indentation data. (G) AFM height image of an apical membrane sheet on a porous substrate. $(H)$ Fluorescence micrograph of apical membrane sheets labelled with Dil(18) on a porous substrate.

membranes for reasons that have been discussed previously. ${ }^{44}$ Force indentation curves were subject to fitting a point-load force model that comprises a linear term associated with prestress of the membrane and a cubic term representing nonlinear membrane theory. ${ }^{45,46}$

$$
F(z)=T_{0} \pi \delta+\frac{\pi \tilde{K}_{\mathrm{A}} \delta^{3}}{3 R^{2}}
$$

Assuming a point-load force rather than computing the contact with a conical indenter is justified since the shape of the indented membrane is indistinguishable from that of the pyramidal indenter (Fig. $3 \mathrm{~F}$ ). The tension $T_{0}$ can be mainly attributed to adhesion of the apical membrane fragment to the pore rims and is therefore not an intrinsic parameter of the cell membrane. Nevertheless, the values are close to the mechanical parameters produced by the actomyosin cortex in living cells. As above, $\tilde{K}_{\mathrm{A}}$ denotes the apparent area compressibility modulus of 
the apical cell membrane. The model is essentially identical to the tension model used to describe indentation of living cells but accounts for the altered geometry of a pore-spanning membrane. Fig. 3E shows two force indentation curves, one obtained from the pore rim (blue, 1) displaying hard-wall repulsion and the other indentation curve recorded on the centre of a pore covered with the apical membrane of confluent MDCK II cells (black, 2). The dotted red line represents a fit according to eqn (8). The corresponding AFM image is shown in Fig. 3G displaying no obvious sign of an actin terminal web. Fluorescence micrographs of apical cell membranes spanning the porous matrix show the presence of protrusions (Fig. $3 \mathrm{H}$ ). We found a mean tension $T_{0}$ of $(0.16 \pm 0.07) \mathrm{mN} \mathrm{m}^{-1}$ and an apparent area compressibility modulus $\tilde{K}_{\mathrm{A}}$ of $(0.009 \pm 0.001) \mathrm{N}$ $\mathrm{m}^{-1}$ representative of a plasma membrane lacking cytoskeleton filaments but associated with a lipid reservoir providing excess membrane area upon stretching the planar fragments.

The value corresponds well to the $\tilde{K}_{\mathrm{A}}$ values (main peak of the bimodal distribution) found for vital MDCK II cells (Fig. 2B) supporting the view that the plasma membrane largely governs the elastic response to a central load at large strains.

We also investigated isolated basolateral membranes spanned over a porous matrix (pore size $1.2 \mu \mathrm{m}$ in diameter) to see whether the apparent area compressibility modulus is different from the apical part of the cell. We found a significantly smaller $\tilde{K}_{\mathrm{A}}$ value of $(0.003 \pm 0.002) \mathrm{N} \mathrm{m}^{-1}$ (Fig. S3 $\dagger$ ) compared to the apical side. We attribute this difference to the presence of caveolae only at the basal side providing additional excess surface area..$^{47,48}$

\subsection{Disrupting the cortical integrity}

So far we could show that the tension model is indenterinvariant and manages to capture the essential physics of indentation experiments. Whether it is sufficient to characterize cell mechanics of confluent monolayers in conjunction with tether pulling experiments will be demonstrated by targeting various structures of the apical membrane.

2.3.1. Impact of cytochalasin D on cellular mechanics. A fully functional actomyosin cortex only maintains tension in the cortex. To quantify the impact of actomyosin integrity on the overall tension, we disrupted the actin cortex by exposing the cell monolayer to cytochalasin $\mathrm{D}(10 \mu \mathrm{M})$ for 15 min promoting depolymerization of the filaments. The change in the cortical structure is visible in the fluorescence micrographs (Fig. 4A-D). When treated with cytochalasin D, F-actin and ezrin distribution is altered, showing a more aggregated and clustered arrangement. As a result, both the overall tension $T_{0}$ and membrane tension $T_{\mathrm{t}}$ drop substantially confirming the crucial role of the actin network for establishing a finite lateral tension in the outer shell (Table 2 and Fig. $4 \mathrm{E}$ and F). The administration of cytochalasin D leads to the disassembly of the F-actin cytoskeleton and enables us to separate the tension generated by the cytoskeleton adhesion to the membrane $\gamma_{\text {ad }}$ and the membrane's in-plane tension $\gamma_{\mathrm{m}}$ from the membrane tension $T_{\mathrm{t}}$ measured by tether pulling (eqn (3)). ${ }^{49}$ After disrupting the cortex integrity the membrane tension $T_{\mathrm{t}}$ is reduced by a factor
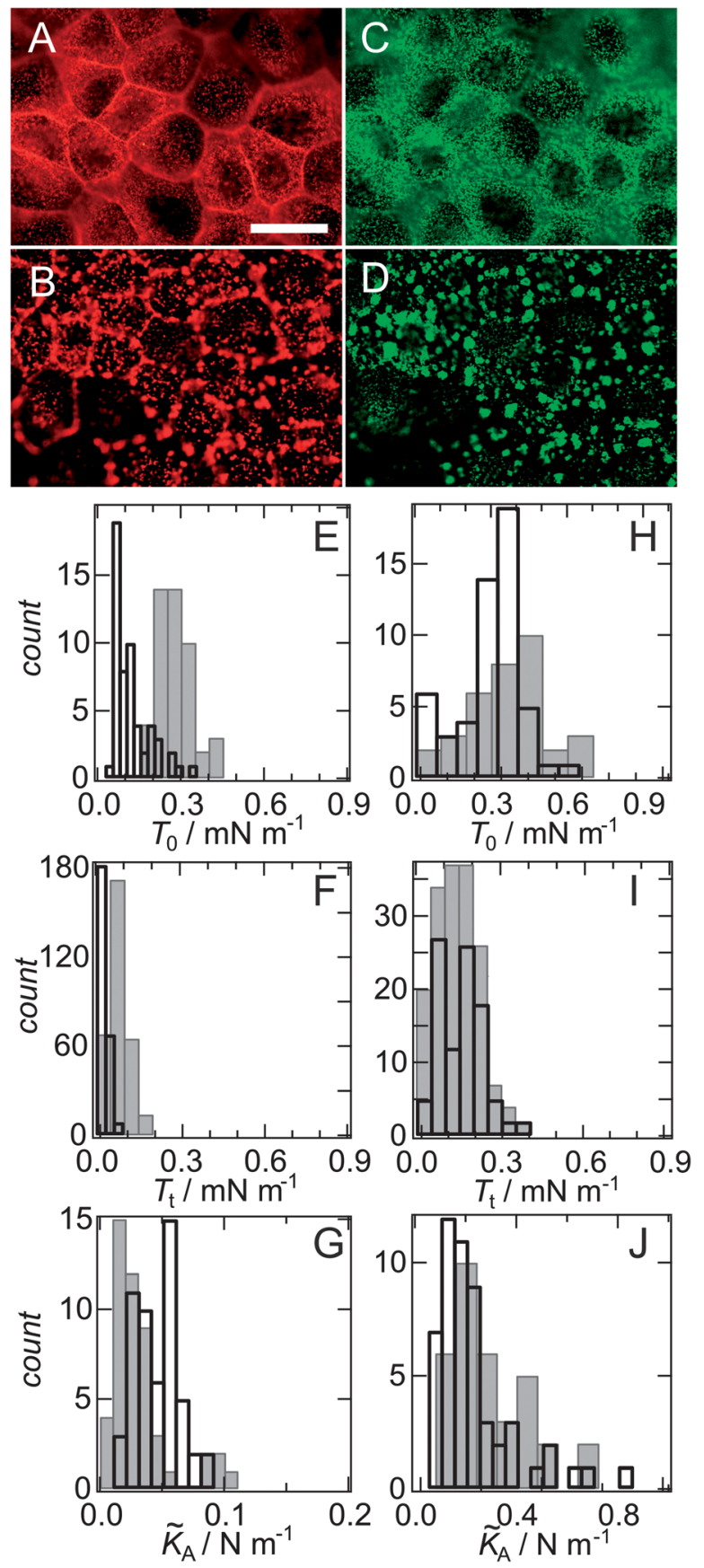

Fig. 4 Fluorescence micrographs of confluent MDCK II cells labelled for F-actin (red, left, Alexa Fluor 546-phalloidin) and ezrin (green, right, Alexa Fluor 488-lgG goat-anti-mouse). Untreated cells ( $A$ and $C$ ) and cytochalasin D treated cells ( $B$ and D). Scale bar: $20 \mu \mathrm{m}$. (E-J) Mechanical investigation of MDCK II cells after disturbing the cortical integrity. Histograms in gray represent parameters prior to addition of chemical stimuli, while transparent bars show data for MDCK II cells after administration of $10 \mu \mathrm{M}$ cytochalasin $\mathrm{D}(\mathrm{E}-\mathrm{G})$ or $50 \mu \mathrm{M}$ blebbistatin $(\mathrm{H}-\mathrm{J})$, respectively. ( $\mathrm{E}$ and $\mathrm{H}$ ) Overall tension $T_{0}$. ( $\mathrm{F}$ and $\mathrm{I}$ ) Histograms of the membrane tension $T_{\mathrm{t}}$. (G and J) Histograms of the apparent area compressibility modulus.

of 2.7 (Table 2). We attribute the remaining value of $0.03 \mathrm{mN}$ $\mathrm{m}^{-1}$ to the membrane's in-plane tension $\gamma_{\mathrm{m}}$, since the cortex which maintains $\gamma_{\text {ad }}$ is destroyed. Thereby $>60 \%$ of membrane tension $T_{\mathrm{t}}$ results from adhesion of the cytoskeleton to the membrane. ${ }^{49}$ 
Table 2 Results from force cycle experiments on confluent MDCK II cell monolayers according to Fig. 4. Data are presented as mean values with standard deviation

\begin{tabular}{llll}
\hline & $T_{0}{ }^{a}\left[\mathrm{mN} \mathrm{m}^{-1}\right]$ & $T_{\mathrm{t}}^{b}\left[\mathrm{mN} \mathrm{m}^{-1}\right]$ & $\tilde{K}_{\mathrm{A}}{ }^{c}\left[\mathrm{~N} \mathrm{~m}^{-1}\right]$ \\
\hline Untreated & $0.28 \pm 0.06$ & $0.08 \pm 0.04$ & $0.19 \pm 0.06$ \\
Cytochalasin D & $0.12 \pm 0.06$ & $0.03 \pm 0.02$ & $0.08 \pm 0.06$
\end{tabular}

${ }^{a}$ Overall tension. ${ }^{b}$ Membrane tension. ${ }^{c}$ Apparent area compressibility modulus.

In contrast, $\tilde{K}_{\mathrm{A}}$ maintains its bimodal distribution centred at $0.02 \mathrm{~N} \mathrm{~m}^{-1}$ and $0.05 \mathrm{~N} \mathrm{~m}^{-1}$ with only a slight shift of maximum II to lower values compared to untreated cells, which might be attributed to a release of membrane area in response to cytoskeleton removal (Fig. 4E-G).

2.3.2. Inhibition of myosin II with blebbistatin. Apart from F-actin severing agents we also used blebbistatin as an inhibitor of myosin II to impede active contraction of the cortex. Myosin II mainly interacts with F-actin bundled as stress fibres in the basal part of the cell. ${ }^{\mathbf{5 0 , 5 1}}$ By varying this force generating arrangement, no changes in the architecture of the cellular apex (ezrin and F-actin distribution (Fig. S1 $\dagger$ )) and measured membrane tension from tether pulling experiments are found (Fig. 4I). However, tremendous structural alterations are observed in the basal part, where huge blebs appear during blebbistatin treatment (Fig. S1†). Blebs are known to nucleate at regions where the membrane cytoskeleton adhesion is weakened. ${ }^{8}$ On the apical side we found no changes in membrane tension $\left(\bar{T}_{\mathrm{t} \text {,untreated }}=(0.15 \pm 0.08) \mathrm{mN} \mathrm{m}^{-1}, \bar{T}_{\mathrm{t}, \mathrm{blebb}}=\right.$ $\left.(0.16 \pm 0.08) \mathrm{mN} \mathrm{m}^{-1}\right)$, while the overall tension $T_{0}$ determined by indentation experiments displays a small but significant decrease $\left(\bar{T}_{0, \text { untreated }}=(0.36 \pm 0.16) \mathrm{mN} \mathrm{m}^{-1}, \bar{T}_{0, \text { blebb }}=(0.29 \pm 0.13) \mathrm{mN}\right.$ $\mathrm{m}^{-1}$, Wilcoxon Rank test, $\left.p=0.04\right)$. This drop in overall tension can be attributed to the loss of active cortical tension generated by disruption of myosin functionality due to blebbistatin treatment. The apparent area compressibility modulus $\tilde{K}_{\mathrm{A}}$ decreases slightly but also significantly from $\tilde{K}_{\mathrm{A} \text {,untreated }}=(0.29 \pm 0.16) \mathrm{N} \mathrm{m}^{-1}$ to $\tilde{K}_{\mathrm{A} \text {,blebb }}=(0.23 \pm 0.16) \mathrm{mN} \mathrm{m}^{-1}$ (Wilcoxon Rank test, $\left.p=0.004\right)$, which reflects an enlargement in the available membrane area due to tension release.

\subsection{Reversible removal of excess membrane reservoirs}

Now we will verify that the apparent area compressibility modulus indeed mirrors the excess surface area stored in membrane reservoirs such as microvilli and investigate how tension evolves if surface area regulation is perturbed. If the low values of the apparent area compressibility modulus $\tilde{K}_{\mathrm{A}}=0.02$ $\mathrm{N} \mathrm{m}^{-1}$ compared to those of a lipid bilayer $\left(K_{\mathrm{A}}=0.1-1 \mathrm{~N} \mathrm{~m}^{-1}\right)$ were a result of excess area stored in microvilli, removal of these reservoirs should consequently lead to an increase in $\tilde{K}_{\mathrm{A}}$ up to the maximal area compressibility modulus $K_{\mathrm{A}}$ of a lipid bilayer. In turn, membrane tension is eventually expected to increase due to lack of membrane reservoirs acting as a buffer to compensate a change in membrane tension.

To verify these assumptions, we added methyl- $\beta$-cyclodextrin (MBCD), which is known to flatten the apical membrane by reducing cholesterol from the plasma membrane. ${ }^{29}$ It has been shown that $80-90 \%$ of total cellular cholesterol can be efficiently extracted from the plasma membranes with MBCD concentrations of 5-10 $\mathrm{mM}$ and exposure times of more than 2 h. ${ }^{29,52-54}$ As first reported by Poole et al. we also found that the number of microvilli is greatly reduced after MBCD treatment (Fig. 5C). ${ }^{29}$

Besides, we observe a time-dependent redistribution of ezrin and F-actin away from the apical plasma membrane (Fig. 5A and B). After $3 \mathrm{~h}$, the cell surface has lost almost all membrane protrusions and appears topographically smooth (Fig. 5C). The overall reduction in the membrane area is corroborated and quantified by impedance analysis of the confluent monolayer (Fig. 5D) revealing that the membrane capacitance $C_{\mathrm{m}}$ decreases by almost $20 \%$ within $3 \mathrm{~h}$ of MBCD treatment $\left(C_{\mathrm{m}}^{\mathrm{oh}}=1.64 \mu \mathrm{F}\right.$ $\mathrm{cm}^{-2}$ and $\left.C_{\mathrm{m}}^{3 \mathrm{~h}}=1.33 \mu \mathrm{F} \mathrm{cm}^{-2}\right)$. Capacitance changes were obtained from ECIS measurements as described previously. ${ }^{55}$ In brief, the complex impedance was measured as a function of frequency and fitted by an appropriate equivalent circuit. ${ }^{56,57}$ At each time point an entire spectrum is used to obtain the capacitance of the cell-layer cultured on the gold electrode (250 $\mu \mathrm{m}$ in diameter). Concomitantly, we found a higher endocytosis rate as a response to cholesterol depletion (Fig. 6), i.e. a larger number of vesicles accumulate in the cytosol.

Importantly, the disappearance of microvilli is fully reversible as shown by monitoring the capacitance and transepithelial resistance of confluent MDCK II cells after removal of cholesterol (Fig. S2 $\dagger$ ). The data prove that cellular viability, membrane integrity, and tight junctions recover $3 \mathrm{~h}$ after MBCD treatment. Although cholesterol depletion is not specific, it affects the cytoskeleton organization as documented in Fig. 5.

Probing the mechanical properties of MBCD treated cells reveals tension values of the apical membrane $\left(T_{0}\right.$ and $\left.T_{\mathrm{t}}\right)$ that continuously increase while reducing the apical excess surface area (Fig. $5 \mathrm{E}-\mathrm{G}$ ). The overall tension $T_{0}$ increases within $3 \mathrm{~h}$ by a factor of 2 , and membrane tension $T_{\mathrm{t}}$ by a factor of 3 . According to eqn (2), it is evident that the active cortical tension $T_{\text {act }}$ stays constant while cholesterol is depleted from the cellular membrane (Fig. 5F). This makes perfect sense since the removal of cholesterol mainly affects the bilayer and the existence of reservoirs but to a much lesser extent the cortical tension. Concomitantly, the apparent area compressibility modulus $\tilde{K}_{\mathrm{A}}$ increases as a result of cholesterol depletion and can be attributed to a decrease of $A_{\mathrm{ex}}$. The removal of the excess surface area stored in membrane protrusions directly leads to changes in the measured apparent area compressibility modulus serving as a reporter for loss and gain of membrane reservoirs. The membrane uptake observed in Fig. 6 (increased endocytotic rate) leads to the measured reduction in the surface area. As a consequence, membrane tension $T_{\mathrm{t}}$ cannot be buffered anymore and thus increases uncontrollably.

Apart from MBCD, dynamin-inhibiting dynasore was used to address the question whether arresting of the endocytosis pathway via clathrin coated pits interferes with surface area regulation. ${ }^{27}$ Since caveolae are largely absent at the apical site of confluent MDCK II cells, ${ }^{48}$ we concentrate on another prominent source of folds, the coated pits, as a possible source 

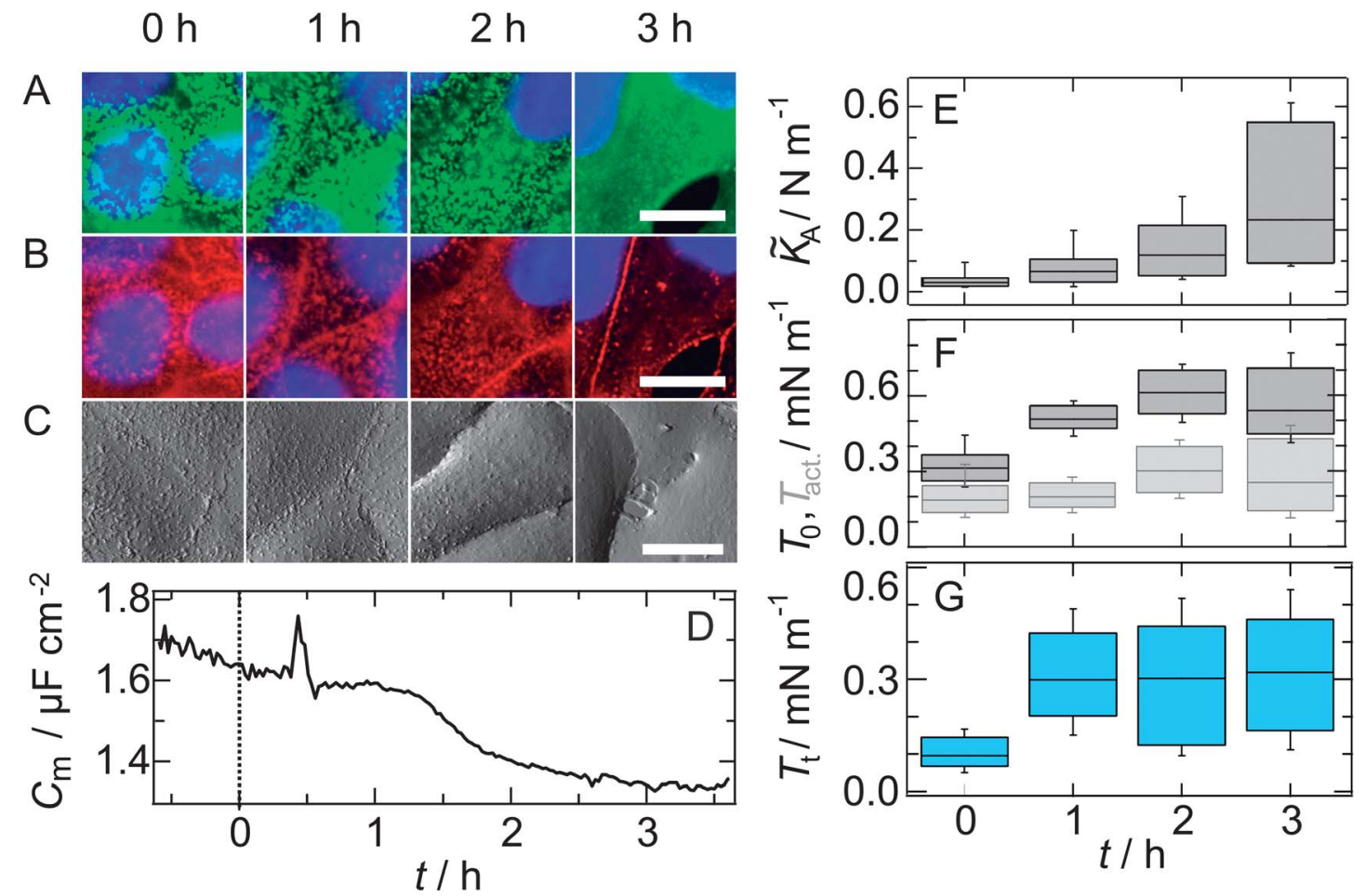

Fig. 5 Effect of cholesterol depletion by MBCD on plasma membrane mechanics of MDCK II cells. Immunostaining of ezrin (green, top, Alexa Fluor 488-IgG goat-antimouse, (A) and F-actin (red, center, Alexa Fluor 546-phalloidin, (B) and corresponding AFM deflection images (C) taken at different exposure times of MBCD. Untreated cells $(0 \mathrm{~h})$ display a large number of microvilli on the plasma membrane, which is reduced after $2 \mathrm{~h}$ of incubation with MBCD. After $3 \mathrm{~h}$ only a few microvilli remain visible at the apical membrane of the cells. Scale bar: $10 \mu \mathrm{m}$. (D) Membrane capacitance $C_{m}$ of MDCK II cells treated with MBCD added at $t=0 \mathrm{~h}$ obtained from fitting timeresolved impedance spectra with an area contact model. (E-G) Mechanical properties (apparent area compressibility modulus $(n=100)$, overall tension $T_{0}(n=100)$, active cortical tension $T_{\text {act }}(n=100)$ and membrane tension $\left.T_{\mathrm{t}}(n=300)\right)$ of MDCK II cells measured at different times after cholesterol depletion of the plasma membrane.
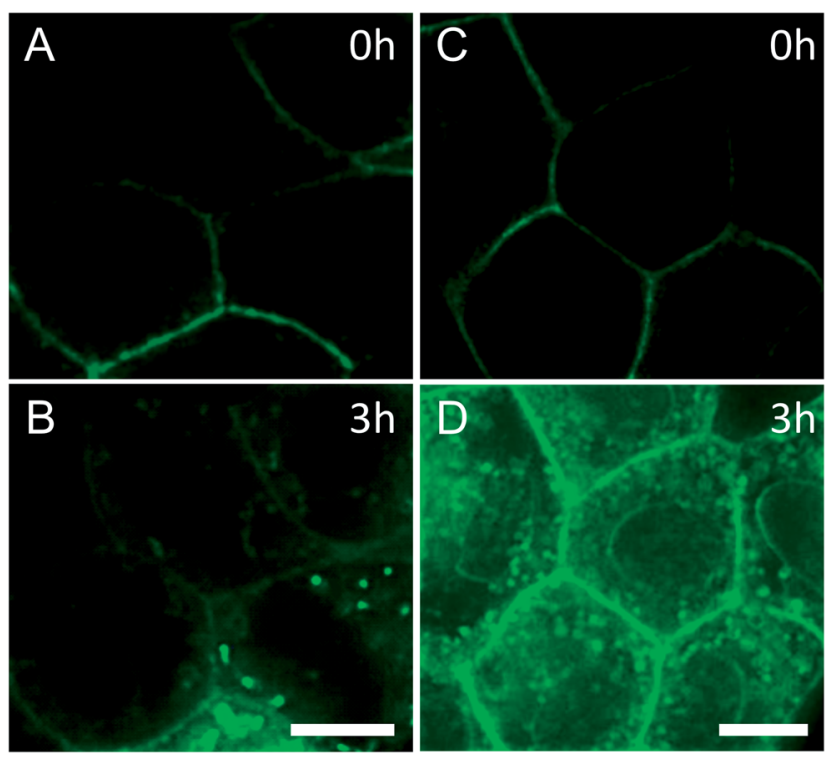

Fig. 6 Membrane staining of living MDCK II cells to visualize membrane uptake after MBCD treatment. The focal plane is in the lower part of the cell (basal). (A and B) Untreated cells showing a normal endocytotic uptake rate. (C and D) Cells before and after $3 \mathrm{~h}$ treatment with MBCD. The green fluorescence displays membrane-uptake during cholesterol depletion; small vesicular structures are detectable in the lower part of the cell (D). of excess surface area. Generally, the impact of dynasore administration on the mechanical parameters is rather small suggesting that other membrane reservoirs play a more important role. We found that the overall tension $T_{0}$ remains after $3 \mathrm{~h}$ of exposure on a constant value $\left(T_{0}^{0 \mathrm{~h}}=0.51 \mathrm{mN} \mathrm{m}^{-1}\right.$, $T_{0}^{3 \mathrm{~h}}=0.49 \mathrm{mN} \mathrm{m}^{-1}$ (median, Fig. 7A)). In contrast the membrane tension $T_{\mathrm{t}}$ slightly increases from $T_{\mathrm{t}}=0.11 \mathrm{mN} \mathrm{m}^{-1}$ to $0.14 \mathrm{mN} \mathrm{m}^{-1}$ (median, Fig. 7B). The apparent area compressibility modulus elevates within $3 \mathrm{~h}$ from $\tilde{K}_{\mathrm{A}}=0.11 \mathrm{~N}$ $\mathrm{m}^{-1}$ to $0.15 \mathrm{~N} \mathrm{~m}^{-1}$ (median, Fig. $7 \mathrm{C}$ ) indicating a decrease in the available surface area. The higher value of $\tilde{K}_{\mathrm{A}}$ implies that the additional surface area cannot by recruited while dynamin is blocked. We propose that excess of plasma membrane stored in clathrin coated pits can no longer participate in surface area regulation thus leading to an increase in $\tilde{K}_{\mathrm{A}}$. Since dynasore extends the lifetime of clathrin coated pits $^{58}$ and does not affect changes in membrane-cytoskeleton attachment sites, it is conceivable that the dynamics of the plasma membrane is slowed down leading to higher membrane in-plane tension $\gamma_{\mathrm{m}}$ and a constant $\gamma_{\text {ad }}$ resulting in a slightly increased membrane tension $T_{\mathrm{t}}$ by following eqn (3), while $T_{0}$ stays constant. Interestingly, the cells maintain their overall tension $T_{0}$ although the available surface area of the plasma membrane is partially lost - it is possible that the contribution of coated pits is simply too small. 

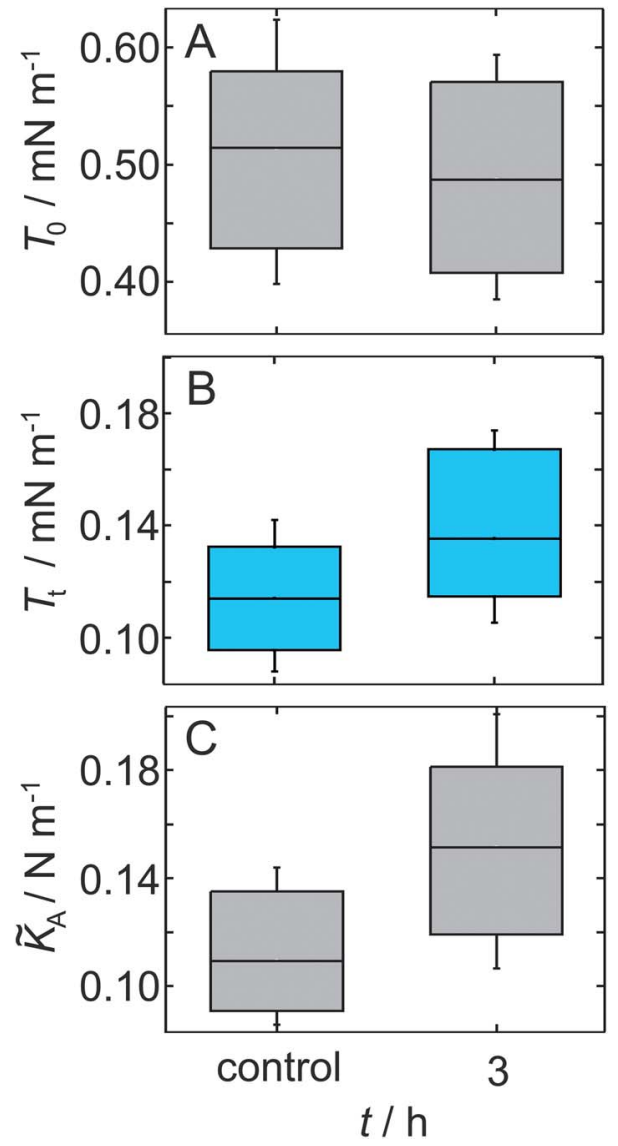

Fig. 7 Mechanical parameters of confluent MDCK II cells after treatment with dynamin-inhibiting dynasore for $3 \mathrm{~h}$. (A) Overall tension $T_{0}$. (B) Membrane tension $T_{\text {t. }}$ (C) Apparent area compressibility modulus $\tilde{K}_{\mathrm{A}}$.

\section{Discussion}

Epithelial cells organized in closed two-dimensional monolayers are frequently confronted with a number of chemical and mechanical challenges comprising toxins and osmotic stress but also morphological transitions during tumorigenesis and wound healing as well as external mechanical deformations. In order to appreciate the elastic response of cells to external stimuli in the context of confluent monolayers appropriate models are needed that include the basic physics of elasticity but remain tractable and cover a large force range.

Here, we could show experimentally that a tension-based model that captures the shell-structure of the apical membrane and has been proven to be largely indenter-invariant can best describe the elastic response of confluent epithelial cells. Essentially, this model considers the cell as a liquid filled bag surrounded by a shell with an isotropic tension. We found that frequently used contact models such as the Hertz-Sneddon model fail to describe indentation of single MDCK II cells in a confluent monolayer especially at large strains at which membrane theory prevails. Precisely, the contact models are unable to follow the experimental at indentation depths beyond $250 \mathrm{~nm}$. Most strikingly, the contact models are inconsistent in terms of Young's modulus if two different indenter geometries and sizes are used to penetrate the same cell. Alarmingly, we found that the Young's modulus of one and the same cell decreases with increasing indenter size by orders of magnitude. In general, contact models based on Hertzian mechanics are by definition only valid for semi-infinite elastic bodies at low indentation depths. Cells display, however, a shell-like structure composed of a plasma membrane associated with the actomyosin cortex. These shells are highly anisotropic and stretching is no longer a second order effect. For instance, membranes are laterally largely inextensible, while bending energy is only a few $k_{\mathrm{B}} T$. As a consequence, bending of shells rules at low indentation depths, while at higher strains lateral dilatation of the shell governs the response to indentation. Pre-stress due to a finite tension stored in the shell is also not included in common contact models. Both bending and pre-stress produce a largely linear force indentation curve, and stretching of the shell laterally, however, generates a nonlinear response - mostly cubic. An indication why contact models are inadequate is the predicted scaling with the radius of the object (the cell). While shell theory predicts that resistance to indentation diminishes with increasing radius, contact mechanical models suggest the opposite. The easiest way to test whether any of the contact models serves to describe the experimental data is to plot forcedistance curves in a $\log$-log plot to confirm the predicted power law scaling. In short, we could not confirm the predicted scaling for our cells (Fig. S5†). Other cell types that are more solid-like might, however, follow these laws.

The two-parameter tension-model initially put forward by Sen et al. and modified by us to match the geometry of confluent polar cells describes the experimental data over the entire force range and produces consistent results in terms of tension and area compressibility modulus independent of the indenter geometry.

We validated our model further by using pore spanning apical membranes transferred from intact living MDCK II cells grown to confluency that are devoid of cytoskeleton filaments and cytosolic components. Adapting the same theory to the different geometrical constraints allowed us to determine the neat area compressibility modulus of apical membranes. We found that the values are essentially identical to those measured on vital MDCK II cells supporting the idea that at large strains membrane mechanics prevails over cortex properties and confirming that a substantial amount of excess surface area is stored.

Altogether, this leaves us with a minimal elastic model that is governed by a constant isotropic tension and an apparent area compressibility modulus giving access to the excess surface area of the plasma membrane and covering extraordinary large strains. The benefit of our approach is that within a single force cycle, encompassing indentation during approach and tether pulling upon retraction, we can determine membrane tension $T_{\mathrm{t}}$, overall tension $T_{0}$, and excess surface area $A_{\mathrm{ex}}$. Using a combination of various chemical stimuli targeting membrane reservoirs, cytoskeleton integrity and contractility we were able to dissect the various tension components in a comprehensive fashion.

The overall tension of cells encompasses cortical tension, i.e. stress in the actomyosin cortex multiplied with thickness and 
in-plane tension in the plasma membrane originating mainly from cytoskeleton-membrane attachment sites. While in many cells cortical tension dominates the cellular response to mechanical challenges over membrane's in-plane tension and elastic contributions, tension homeostasis of the plasma membrane plays an important role in adhesion, spreading and migration of cells. ${ }^{17,59}$ It was the central question to what extent the plasma membrane governs cellular mechanics of epithelial cells and how surface area regulation maintains a constant tension in the context of a confluent monolayer.

In untreated cells we found that membrane tension $\left(T_{\mathrm{t}}=0.1\right.$ $\mathrm{mN} \mathrm{m}^{-1}$ ) provides a significant contribution to the overall tension $\left(T_{0}=0.3 \mathrm{mN} \mathrm{m}^{-1}\right)$ and cannot - as frequently justified for other cell types - be neglected. ${ }^{36,60}$ The membrane tension of the confluent cells is substantial, in contrast to the membrane tension found for adherent motile cells ranging from $0.003 \mathrm{mN}$ $\mathrm{m}^{-1}$ in neuronal growth cones ${ }^{42}$ to $0.05 \mathrm{mN} \mathrm{m}^{-1}$ in melanoma cells. ${ }^{37}$ Fibroblasts, for instance, generate a considerably larger cortical tension $\left(T_{\mathrm{act}}=2 \mathrm{mN} \mathrm{m}^{-1}\right)$ in comparison to membrane tension $\left(T_{\mathrm{t}}=0.1 \mathrm{mN} \mathrm{m}^{-1}\right)$.

Our experiments show that membrane tension mainly originates from attachment sites of the membrane to the actin network via ERM proteins as shown by immunostaining of ezrin and co-localization with actin filaments (Fig. 1A and 4A-D). Our first experiment targets the integrity of the actin shell using cytochalasin D. Immediately after administration of the actinsevering agent both the overall tension $T_{0}$ (comprising the cortical tension $T_{\text {act }}$ ) and membrane tension $T_{\mathrm{t}}$, essentially $\gamma_{\mathrm{ad}}$, drop considerably, which supports the idea that the actin network is mainly responsible for cortical tension and membrane tension. Destroying the actin network therefore releases the cortical tension and removes attachment sites of the plasma membrane. Consequently, the remaining membrane tension of only $0.03 \mathrm{mN}$ $\mathrm{m}^{-1}$ is attributed to the in-plane tension of the membrane $\gamma_{\mathrm{m}}$, which is not negligible as for instance in mesenchymal cells such as fibroblasts. Therefore, we can conclude that for vital confluent MDCK II cells the dominating source of tension parts into membrane-cytoskeleton attachment sites and the actomyosin cortex itself. This finding is further backed up by administration of blebbistatin that only releases cortical tension but keeps the membrane tension untouched. The removal of cholesterol from the plasma membrane results in an uncontrolled increase of membrane tension, while cortical tension stays constant. The loss of reservoirs due to removal of cholesterol prevents the cells from buffering tension effectively and is therefore responsible for the increase in $T_{\mathrm{t}}$.

As far as the excess area is concerned we found that $\tilde{K}_{\mathrm{A}}$ of untreated MDCK II cells displays a bimodal distribution centering on a low (peak I, $0.02 \mathrm{~N} \mathrm{~m}^{-1}$ ) and a high (peak II, $0.1 \mathrm{~N}$ $\left.\mathrm{m}^{-1}\right) \tilde{K}_{\mathrm{A}}$ value, respectively. This distribution might reflect two distinct topographical regions on the apical cell membrane. One region is associated with an extended membrane reservoir, giving rise to low $\tilde{K}_{\mathrm{A}}$ values and a second population, in which the membrane constitutes a thin stretched shell that shows an area compressibility modulus resembling those of artificial lipid bilayers or membranes from red blood cells. ${ }^{61-63}$ It is commonly accepted that $K_{\mathrm{A}}$ values of pure lipid bilayers and cell membranes are in the range of $0.1-1 \mathrm{~N} \mathrm{~m}^{-1}$ depending on the lipid composition, i.e. the presence of cholesterol increases this value substantially. ${ }^{\mathbf{3 0 , 6 1}}$ Significantly lower $\tilde{K}_{\mathrm{A}}$-values are therefore indicative of the excess surface area stored in the highly roughened surface of the apical membrane encompassing protrusions such as microvilli (Fig. 1B). ${ }^{64}$ Butor and Davoust quantified this excess area or membrane folding of MDCK II cells using electron microscopy. They found that MDCK II cells display a folding factor of $\sim 8$ for the apical plasma membrane, ${ }^{65}$ which is in good agreement with our findings from indentation experiments (folding factor of $\sim 5$ following eqn (4)). This implies that lysis tension (tension at which the membrane ruptures) of $10-20 \mathrm{mN} \mathrm{m}^{-1}$ requires extremely large strains.

Administration of cytochalasin D results in a slight lowering of peak II, while peak I representing lower $\tilde{K}_{\mathrm{A}}$ values remains unaffected. Hence, we can attribute the lower $\tilde{K}_{\mathrm{A}}$ values, of untreated MDCK II cells, to areas on the cell membrane with no or little adhesive support and therefore accessibility to the excess surface area. If in-plane stretching of the membrane is indeed compensated by drawing an additional membrane from a highly dynamic surface area reservoir, ${ }^{66}$ a stimulated loss of these reservoirs should lead to a larger overall tension and disappearance of low $\tilde{K}_{\mathrm{A}}$ values. A way to test this hypothesis was to remove cholesterol from the plasma membrane. Cholesterol depletion leads to dispersion of lipid rafts and therefore to removal of microvilli acting as potential surface area reservoirs for the cell membrane. ${ }^{28,29}$ Indeed, we detect an increased endocytosis rate, i.e. vesicles accumulated in the cytosol (Fig. 6) in response to cholesterol depletion. The reduction of membrane reservoirs clearly results in a significant increase in the membrane tension $\left(T_{0}\right.$ and $T_{\mathrm{t}}$ ) by a factor of two and an increase in $\tilde{K}_{\mathrm{A}}$ documenting a loss of excess surface area. In support of this finding we monitored the reduction of membrane area by measuring time resolved changes in the membrane capacitance of a confluent cell monolayer. The reduction in the membrane area shifts the apparent area compressibility modulus $\tilde{K}_{\mathrm{A}}$ to higher values, almost by a factor of five.

Altogether, we can show that the elastic response of confluent epithelial cells is governed by an intricate interplay between cytoskeleton-membrane linkage generating membrane tension that is buffered by a surface area reservoir of membrane folds and an active actomyosin cortex that produces a cortical tension to resist cellular deformation. While the elastic response of many cell types is governed by cortical tension produced by the active actomyosin cortex, the mechanical response of epithelial cells in the context of a confluent monolayer is largely influenced also by membrane tension produced by the cytoskeleton adhering to the plasma membrane. It is evident that an excess of surface area is required by the cell to counteract changes in membrane tension to avoid lysis. Similarly, Knutton et al. and Erickson et al. showed that cells in interphase and mitosis exhibit a continuum of micro-folds and invaginations ${ }^{\mathbf{6 4 , 6 7}}$ that suggests the presence of membrane reservoirs to respond to tension changes. The fivefold excess in the surface area is typical as reported by Butor and Davoust ${ }^{65}$ and allows the cells to quickly respond to changes in membrane tension. 
While cortical tension dominates the elastic response at low strains, the response of the apical membrane is entirely governed by the area compressibility of the lipid membrane. Notably, the cortex alone possesses a considerably smaller area compressibility modulus at least by two orders of magnitude, rendering the plasma membrane the dominating cause for resisting mechanical challenges at larger strains.

The inextensibility of the lipid bilayer, which is the cause for the large area compressibility modulus $\left(>0.1 \mathrm{~N} \mathrm{~m}^{-1}\right)$, requires the cell to regulate the surface area in order to accommodate large strains. Bending stiffness of the cortex does not seem to play an important role in the mechanics of adherent polar epithelial cells in the context of a confluent monolayer since the cortex is too thin and not stiff enough. In summary, the experiments allow us to draw a comprehensive picture of how surface area regulation is reflected in membrane mechanics.

\section{Experimental section}

\section{Cell culture}

MDCK II cells, obtained from the Health Protection Agency, Salisbury, UK, were maintained in minimal essential medium (MEM) with Earle's salts and $2.2 \mathrm{~g} \mathrm{~L}^{-1} \mathrm{NaHCO}_{3}$ (Biochrom, Berlin, Germany) supplemented with $4 \mathrm{mM}$ L-glutamine (Biochrom, Berlin, Germany), $0.2 \mathrm{mg} \mathrm{mL}^{-1}$ penicillin-streptomycin (PAA, Pasching, Austria) and 10\% FCS (fetal calf serum, PAA, Pasching, Austria) at $37{ }^{\circ} \mathrm{C}$ in a $5 \% \mathrm{CO}_{2}$ humidified incubator. Confluent cells were released with trypsin/EDTA $(0.5 \% / 0.2 \%)$ (Biochrom, Berlin, Germany) and subcultured weekly.

\section{Preparation of apical and basolateral membrane sheets}

Porous silicon chips were obtained from fluXXion B.V (Eindhoven, NL). We used $5 \times 5 \mathrm{~mm}^{2}$ sized chips with a pore diameter of $1.2 \mu \mathrm{m}$. Confluent MDCK II cells were washed with PBS (phosphate buffered saline, Biochrom, Berlin, Germany) and incubated with distilled water for $3 \mathrm{~min}$. To enhance electrostatic adhesion between the substrate and the membranes, the chips were incubated with $1 \mathrm{mg} \mathrm{mL}^{-1}$ poly-D-lysine (Sigma-Aldrich, Steinheim, Germany, MW = 70 000-150 000) solution for $10 \mathrm{~min}$ followed by thorough rinsing with ultrapure water. The chips were gently pressed on the cells' apical surface for $20 \mathrm{~min}$ to form a conformal surface contact. By lifting off the chips from the cells, the apical membranes were transferred to the poly-D-lysine coated chips with their cytoplasmic side facing up. For this Experimental section, the MFP-3D (Asylum Research, Santa Barbara, USA) was used both for imaging and force spectroscopy.

Basolateral membranes were prepared on the porous chips following the protocol developed by Lorenz et al. ${ }^{68}$

\section{Disrupting cortex integrity}

We disrupted the actin cortex by exposing the cell monolayer of confluent MDCK II cells to cytochalasin D (Sigma-Aldrich, Steinheim, Germany) $(10 \mu \mathrm{M})$ for $15 \mathrm{~min}$. We examined at least 6 cells and analysed 50 representative force curves out of 400 recorded for indentation and 320 for tether pulling. To examine to what extent the acto-myosin connection affects the mechanical properties of the plasma membrane of MDCK II cells, blebbistatin (Sigma-Aldrich, Steinheim, Germany) is added in a final concentration of $50 \mu \mathrm{M}$ and incubated for 30 min. We examined 7 cells and analysed 50 representative force curves out of 300 recorded ones for indentation and 115 for tether pulling, respectively.

\section{Removal of excess membrane reservoirs}

We removed excess membrane from the cells by exposing confluent MDCK II monolayers to cholesterol depleting methyl$\beta$-cyclodextrin (MBCD, Sigma-Aldrich, Steinheim, Germany) (10 $\mu \mathrm{M})$ for $1-3 \mathrm{~h}$.

We blocked endocytosis of confluent MDCK II cells by hindering dynamin with dynasore hydrate (Sigma-Aldrich, Steinheim, Germany) $(100 \mu \mathrm{M})$ and recorded force curves after 3 $\mathrm{h}$ of exposure. We examined at least 5 cells and analysed 70 representative force curves out of 500 recorded and 185 for tether pulling.

\section{Electric cell-substrate impedance sensing}

Electric cell-substrate impedance sensing (ECIS) measures the complex impedance of cells cultured on ultrasmall gold electrodes, which allows monitoring morphological changes of cells induced by biological, chemical or physical stimuli in real time. ${ }^{56,69}$ ECIS measurements were carried out using 400000 MDCK II cells seeded onto gold electrodes ( $250 \mu \mathrm{m}$ in diameter) of $8 \mathrm{~W} 1 \mathrm{E}$ arrays (Ibidi, Martinsried, Germany) and stored in a humidified incubator set to $37{ }^{\circ} \mathrm{C}$ and $5 \% \mathrm{CO}_{2}$. After a time interval of $24 \mathrm{~h}$, sufficient for adhesion and spreading of the cells, the cell layer reached confluency and was subsequently treated with MBCD (methyl- $\beta$-cyclodextrin, Sigma-Aldrich, Steinheim, Germany) in a final concentration of $10 \mathrm{mM}$. Timeresolved frequency scans (impedance spectra) were acquired with an ECIS Ztheta-setup (Applied Biophysics, Troy, NY). Frequency sweeps were subjected to complex fitting employing an area contact model of Lo and Ferrier, ${ }^{57}$ resulting in time resolved parameters $R_{\mathrm{b}}$ reflecting the transepithelial resistance, $\alpha$ being related to the cell-substrate distance $\left(\alpha \propto \sqrt{h^{-1}}\right)$, and $C_{\mathrm{m}}$ mirroring the membrane capacitance of the cells.

\section{Cell labeling}

Cells were grown in Ibidi ${ }^{\circledR} \mu$-dishes (Ibidi, Martinsried, Germany) to confluency and treated either with $10 \mathrm{mM}$ methyl- $\beta$-cyclodextrin (Sigma-Aldrich, Steinheim, Germany) for 1-3 h, cytochalasin D (Sigma-Aldrich, Steinheim, Germany) $(10 \mu \mathrm{M})$ for $15 \mathrm{~min}$ or blebbistatin (Sigma-Aldrich, Steinheim, Germany) $(50 \mu \mathrm{M})$ for 30 min. Cells were fixed with $4 \%$ paraformaldehyde (PFA) in PBS for $20 \mathrm{~min}$. Dyes and antibodies were diluted in dilution buffer (1\% (w/v) BSA and $0.3 \%(\mathrm{v} / \mathrm{v})$ Triton-X 100 in PBS), which also permeabilises the cells. F-actin labelling was carried out with Alexa Fluor 546-phalloidin (Invitrogen, Darmstadt, Germany, diluted as recommended by the manufacturer, $5 \mu \mathrm{L}$ methanolic stock solution with $200 \mu \mathrm{L}$ dilution buffer for each coverslip). For ezrin labelling, cells were incubated with primary monoclonal antibodies (IgG anti-mouse, BD Biosciences, Heidelberg, Germany) (4 $\mu \mathrm{g} \mathrm{mL}^{-1}$ in dilution buffer) for $1 \mathrm{~h}$ at room 
temperature and subsequently labelled with secondary Alexa Fluor 488-conjugated goat-anti-mouse IgG1 antibodies (Invitrogen, Darmstadt, Germany) for $45 \mathrm{~min}\left(4 \mu \mathrm{g} \mathrm{mL}{ }^{-1}\right.$ in dilution buffer). Membrane labelling of living cells was carried out by incubating cells for $1 \mathrm{~min}$ in PKH2-linker dye (5 $\mu \mathrm{M}$ in PBS, Sigma-Aldrich, Steinheim, Germany). After incubation, cells are rinsed with warm HEPES buffered MEM-medium and mounted under the microscope under temperature control $\left(37^{\circ} \mathrm{C}\right)$. Cells are observed with and without the influence of MBCD.

\section{Atomic force microscopy for imaging}

AFM imaging was performed with a Nanowizard® ${ }^{\circledR}$ II AFM (JPK Instruments AG, Berlin, Germany) mounted on an Olympus IX 81 inverted light microscope. The lateral resolution increases with smaller contact area between the tip and sample. Consequently, fixation of cells increases the stiffness of the sample and concomitantly also reduces the contact area. Therefore, cells were fixated using $2.5 \%$ glutardialdehyde in PBS for 20 min. Silicon nitride cantilevers (MLCT, Bruker AFM Probes, Camarillo, USA) with a nominal force constant of $0.01 \mathrm{~N} \mathrm{~m}^{-1}$ were employed. Cells were imaged in PBS at room temperature with a scan rate of $0.2 \mathrm{~Hz}$ and processed with JPK Image Processing ${ }^{\circledR}$ software (JPK Instruments AG, Berlin, Germany).

\section{Atomic force microscopy for elasticity measurements}

Force curves were taken continuously on a Nanowizard ${ }^{\circledR}$ II AFM (JPK instruments AG, Berlin, Germany) while scanning laterally across the sample referred to as force mapping with a constant force for all performed experiments of $1 \mathrm{nN} .^{70,71}$ MDCK II cells seeded on Ibidi ${ }^{\circledR} \mu$-dishes (Ibidi, Martinsried, Germany) and grown to confluency were mounted on a PetriDishHeater ${ }^{\circledR}$ (JPK instruments AG, Berlin, Germany) set to 37 ${ }^{\circ} \mathrm{C}$ with HEPES buffered culture medium. Force curves were processed by applying an extended tension model first described by Sen et al. that we extended to accommodate cellular geometry of confluent cells. ${ }^{32}$ The average radius of the apical cap protruding from the cell layer was found to be $11 \mu \mathrm{m}$ (Fig. 1C). The corresponding contact angle of the spherical cap measured by AFM imaging was found to be $20^{\circ}$. The height of the cellular apex and therefore the modelled spherical cap was found to be $1 \mu \mathrm{m}$.

\section{Atomic force microscopy for probing tether forces}

Prior to tether pulling, cantilevers (MLCT, Bruker AFM Probes, Camarillo, USA) were plasma cleaned for $30 \mathrm{~s}$ (Argon) and incubated with $2.5 \mathrm{mg} \mathrm{mL}^{-1}$ concanavalin A - FITC conjugated (Sigma-Aldrich, Steinheim, Germany) - in PBS for $1.5 \mathrm{~h}$ to establish a strong contact with the apical surface. Both the approach and retrace velocities were set to $2 \mu \mathrm{m} \mathrm{s}{ }^{-1}$. Tether forces were determined from force distance curves (Fig. 1D) and analysed using eqn (5).

\section{Conclusions}

The apical membrane of epithelial cells in the context of confluent monolayers resists external forces by a static tension comprising membrane tension due to attachment to the cytoskeleton and cortical tension generated by an active actomyosin shell. We assume that the tension of the membrane changes linearly with variation in its surface area according to a $2 \mathrm{D}$ Hookean spring. For confluent cells, contact models based on a single elastic modulus are largely inadequate to describe the elastic response to indentation since the results show a significant dependency on the indenter geometry.

With a single force cycle encompassing indentation followed by tether pulling upon retraction we are able to gain access to all three parameters, cortical tension, membrane tension, and excess membrane area, simultaneously. Interfering with cortical integrity and surface area regulation to buffer tension changes in combination with mechanical probing of isolated, pore spanning apical membranes draws a comprehensive picture of how cells respond to external mechanical stress. We found that dissolution of the actin cortex results in a drop of cortical tension and membrane tension due to loss of F-actinmembrane attachment sites that cannot be buffered by surface area regulation. In contrast, removing excess membrane area stored in microvilli results in an increase of membrane tension that cannot be compensated by sacrificing existing reservoirs. The ability of a cell to perform significant morphological changes as required during cell migration and division requires unperturbed access to the excess area stored in membrane folds. The findings are backed up by data from isolated membrane sheets derived from the apical side and transferred to a porous substrate. Interestingly, opposed to mesenchymal cells or neutrophils, membrane tension of epithelial cells is of the same order as cortical tension thus playing a more important role in the cells' elastic response.

\section{Notes and references}

1 P. A. Janmey and C. A. McCulloch, Annu. Rev. Biomed. Eng., 2007, 9, 1-34.

2 K. E. Kasza, A. C. Rowat, J. Liu, T. E. Angelini, C. P. Brangwynne, G. H. Koenderink and D. A. Weitz, Curr. Opin. Cell Biol., 2007, 19, 101-107.

3 S. Suresh, Acta Mater., 2007, 55, 3989-4014.

4 H. Hertz, J. Reine Angew. Math., 1881, 92, 156-171.

5 A. B. Mathur, A. M. Collinsworth, W. M. Reichert, W. E. Kraus and G. A. Truskey, J. Biomech., 2001, 34, 1545-1553.

6 K. D. Costa and F. C. P. Yin, J. Biomech. Eng., 1999, 121, 462471.

7 R. G. Fehon, A. I. McClatchey and A. Bretscher, Nat. Rev. Mol. Cell Biol., 2010, 11, 276-287.

8 J.-Y. Tinevez, U. Schulze, G. Salbreux, J. Roensch, J.-F. Joanny and E. Paluch, Proc. Natl. Acad. Sci. U. S. A., 2009, 106, 1858118586.

9 E. Paluch, C. Sykes, J. Prost and M. Bornens, Trends Cell Biol., 2006, 16, 5-10.

10 G. Charras and E. Paluch, Nat. Rev. Mol. Cell Biol., 2008, 9, 730-736.

11 M. P. Sheetz and J. Dai, Trends Cell Biol., 1996, 6, 85-89.

12 D. Raucher and M. P. Sheetz, J. Cell Biol., 1999, 144, 497-506. 
13 J. Dai, H. P. Ting-Beall and M. P. Sheetz, J. Gen. Physiol., 1997, 110, 1-10.

14 G. Apodaca, Am. J. Physiol. Ren. Physiol., 2002, 282, 179-190.

15 T. Togo, T. B. Krasieva and R. A. Steinhardt, Mol. Biol. Cell, 2000, 11, 4339-4346.

16 A. Pietuch, B. R. Brückner and A. Janshoff, Biochim. Biophys. Acta, Mol. Cell Res., 2013, 1833, 712-722.

17 N. C. Gauthier, M. A. Fardin, P. Roca-Cusachs and M. P. Sheetz, Proc. Natl. Acad. Sci. U. S. A., 2011, 108, 14467-14472.

18 D. Raucher and M. P. Sheetz, J. Cell Biol., 2000, 148, 127-136.

19 A. Pietuch and A. Janshoff, Open Biol., 2013, 3, 130084.

20 C. E. Morris and U. Homann, J. Membr. Biol., 2001, 179, 79102.

21 H. Thorn, K. G. Stenkula, M. Karlsson, U. Ortegren, F. H. Nystrom, J. Gustavsson and P. Stralfors, Mol. Biol. Cell, 2003, 14, 3967-3976.

22 L. Kozera, E. White and S. Calaghan, PLoS One, 2009, 4, e8312.

23 J. Martens and M. Radmacher, Pflugers Arch., 2008, 456, 95100.

24 A. F. Straight, A. Cheung, J. Limouze, I. Chen, N. J. Westwood, J. R. Sellers and T. J. Mitchison, Science, 2003, 299, 1743-1747.

25 C. Rotsch and M. Radmacher, Biophys. J., 2000, 78, 520-535.

26 J. F. Casella, M. D. Flanagan and S. Lin, Nature, 1981, 293, 302-305.

27 E. Macia, M. Ehrlich, R. Massol, E. Boucrot, C. Brunner and T. Kirchhausen, Dev. Cell, 2006, 10, 839-850.

28 L. J. Pike and J. M. Miller, J. Biol. Chem., 1998, 273, 2229822304.

29 K. Poole, D. Meder, K. Simons and D. Müller, FEBS Lett., 2004, 565, 53-58.

30 O. P. Hamill and B. Martinac, Physiol. Rev., 2001, 81, 685-740.

31 M. Krieg, Y. Arboleda-Estudillo, P. H. Puech, J. Kafer, F. Graner, D. J. Muller and C. P. Heisenberg, Nat. Cell Biol., 2008, 10, 429-436.

32 S. Sen, S. Subramanian and D. E. Discher, Biophys. J., 2005, 89, 3203-3213.

33 A. A. Boulbitch, Phys. Rev. E: Stat. Phys., Plasmas, Fluids, Relat. Interdiscip. Top., 1998, 57, 2123-2128.

34 I. J. Goldstein and R. N. Iyer, Biochim. Biophys. Acta, 1966, 121, 197-200.

35 M. P. Sheetz, Nat. Rev. Mol. Cell Biol., 2001, 2, 392-396.

36 R. M. Hochmuth and W. D. Marcus, Biophys. J., 2002, 82, 2964-2969.

37 J. Dai and M. P. Sheetz, Biophys. J., 1999, 77, 3363-3370.

38 F. Brochard-Wyart, N. Borghi, D. Cuvelier and P. Nassoy, Proc. Natl. Acad. Sci. U. S. A., 2006, 103, 7660-7663.

39 M. Krieg, J. Helenius, C. P. Heisenberg and D. J. Muller, Angew. Chem., Int. Ed., 2008, 47, 9775-9777.

40 I. N. Sneddon, Int. J. Eng. Sci., 1965, 3, 47-57.

41 P. Carl and H. Schillers, Pflugers Arch., 2008, 457, 551-559.

42 F. M. Hochmuth, J. Y. Shao, J. Dai and M. P. Sheetz, Biophys. J., 1996, 70, 358-369.
43 T. Fine, I. Mey, C. Rommel, J. Wegener, C. Steinem and A. Janshoff, Soft Matter, 2009, 5, 3262-3265.

44 M. Kocun and A. Janshoff, Small, 2012, 8, 847-851.

45 U. Komaragiri, M. R. Begley and J. G. Simmonds, J. Appl. Mech., 2005, 72, 203-212.

46 C. Lee, X. Wei, J. W. Kysar and J. Hone, Science, 2008, 321, 385-388.

47 U. Vogel, K. Sandvig and B. van Deurs, J. Cell Sci., 1998, 111, 825-832.

48 U. Lahtinen, M. Honsho, R. G. Parton, K. Simons and P. Verkade, FEBS Lett., 2003, 538, 85-88.

49 M. P. Sheetz, Nat. Rev. Mol. Cell Biol., 2001, 2, 392-396.

50 A. Castillo, R. Lagunes, M. Urbán, E. Frixione and I. Meza, J. Muscle Res. Cell Motil., 1998, 19, 557-574.

51 T. A. Sutton, H. E. Mang and S. J. Atkinson, Am. J. Physiol. Renal Physiol., 2001, 281, F810-F818.

52 R. Zidovetzki and I. Levitan, Biochim. Biophys. Acta, Biomembr., 2007, 1768, 1311-1324.

53 K. Dreja, M. Voldstedlund, J. Vinten, J. Tranum-Jensen, P. Hellstrand and K. Swärd, Arterioscler., Thromb., Vasc. Biol., 2002, 22, 1267-1272.

54 M. Edidin, Annu. Rev. Biophys. Biomol. Struct., 2003, 32, 257-283. 55 M. Tarantola, A. Pietuch, D. Schneider, J. Rother, E. Sunnick, C. Rosman, S. Pierrat, C. Sonnichsen, J. Wegener and A. Janshoff, Nanotoxicology, 2011, 5, 254-268.

56 I. Giaever and C. R. Keese, Nature, 1993, 366, 591-592.

57 C.-M. Lo and J. Ferrier, Phys. Rev. E: Stat. Phys., Plasmas, Fluids, Relat. Interdiscip. Top., 1998, 57, 6982-6987.

58 D. Loerke, M. Mettlen, D. Yarar, K. Jaqaman, H. Jaqaman, G. Danuser and S. L. Schmid, PLoS Biol., 2009, 7, e1000057. 59 G. Salbreux, G. Charras and E. Paluch, Trends Cell Biol., 2012, 22(10), 536-545.

60 J. Y. Shao and R. M. Hochmuth, Biophys. J., 1996, 71, 2892-2901. 61 W. Rawicz, B. A. Smith, T. J. McIntosh, S. A. Simon and E. Evans, Biophys. J., 2008, 94, 4725-4736.

62 E. A. Evans, R. Waugh and L. Melnik, Biophys. J., 1976, 16, 585-595.

63 B. Daily, E. L. Elson and G. I. Zahalak, Biophys. J., 1984, 45, 671-682.

64 S. Knutton, M. C. Sumner and C. A. Pasternak, J. Cell Biol., 1975, 66, 568-576.

65 C. Butor and J. Davoust, Exp. Cell Res., 1992, 203, 115-127. 66 P. Sens and M. S. Turner, Phys. Rev. E: Stat., Nonlinear, Soft Matter Phys., 2006, 73, 031918.

67 C. A. Erickson and J. P. Trinkaus, Exp. Cell Res., 1976, 99, 375-384.

68 B. Lorenz, I. Mey, S. Steltenkamp, T. Fine, C. Rommel, M. M. Müller, A. Maiwald, J. Wegener, C. Steinem and A. Janshoff, Small, 2009, 5, 832-838.

69 C. Xiao and J. H. T. Luong, Biotechnol. Prog., 2003, 19, 1000-1005. 70 M. Radmacher, M. Fritz, C. M. Kacher, J. P. Cleveland and P. K. Hansma, Biophys. J., 1996, 70, 556-567.

71 H. Haga, S. Sasaki, K. Kawabata, E. Ito, T. Ushiki and T. Sambongi, Ultramicroscopy, 2000, 82, 253-258. 